\title{
HARDY SPACES ON WEIGHTED HOMOGENEOUS TREES
}

\author{
LAURA ARDITTI, ANITA TABACCO AMND MARIA VALLARINO
}

\begin{abstract}
Aвstract. We consider an infinite homogeneous tree $\mathscr{V}$ endowed with the usual metric $d$ defined on graphs and a weighted measure $\mu$. The metric measure space $(\mathscr{V}, d, \mu)$ is nondoubling and of exponential growth, hence the classical theory of Hardy spaces does not apply in this setting. We construct an atomic Hardy space $H^{1}(\mu)$ on $(\mathscr{V}, d, \mu)$ and investigate some of its properties, focusing in particular on real interpolation properties and on boundedness of singular integrals on $H^{1}(\mu)$.
\end{abstract}

\section{InTRODUCTION}

Let $\mathscr{V}$ be an infinite homogeneous tree of order $q+1$ endowed with the usual distance $d$ defined on a graph (see Section 2 for the precise definitions). Fix a doubly-infinite geodesic $g$ in $\mathscr{V}$ and define a mapping $N: g \rightarrow \mathbb{Z}$ such that

$$
|N(x)-N(y)|=d(x, y) \quad \forall x, y \in g .
$$

We define the level function $\ell: \mathscr{V} \rightarrow \mathbb{Z}$ as

$$
\ell(x)=N\left(x^{\prime}\right)-d\left(x, x^{\prime}\right),
$$

where $x^{\prime}$ is the only vertex in $g$ such that $d\left(x, x^{\prime}\right)=\min \{d(x, z): z \in g\}$. Let $\mu$ be the measure on $\mathscr{V}$ defined by

$$
\int_{\mathscr{V}} f d \mu=\sum_{x \in \mathscr{V}} f(x) q^{\ell(x)}
$$

for every function $f$ defined on $\mathscr{V}$. Then $\mu$ is a weighted counting measure. We shall show in Subsection 2.1 that the space $(\mathscr{V}, d, \mu)$ is nondoubling and it is of exponential growth. In particular on such space the classical Calderón-Zygmund theory does not hold.

Hebisch and Steger [8] developed a new Calderón-Zygmund theory which can be applied also to nondoubling metric measure spaces and showed that such a theory can be applied to the space $(\mathscr{V}, d, \mu)$. In particular they proved that there exists a family of appropriate sets in $\mathscr{V}$, which are called Calderón-Zygmund sets, which replace the family of balls in the classical Calderón-Zygmund theory. We mention also that some properties of the space $(\mathscr{V}, d, \mu)$ were investigated in more detail in [1].

Key words and phrases. Hardy spaces; homogeneous trees; exponential growth.

The second and third authors are members of the Gruppo Nazionale per l'Analisi Matematica, la Probabilità e le loro Applicazioni (GNAMPA) of the Istituto Nazionale di Alta Matematica (INdAM). . 
The purpose of this work is to develop a theory of Hardy spaces on $(\mathscr{V}, d, \mu)$, which is a natural development of the Calderón-Zygmund theory introduced in [8]. Following the classical atomic definition of Hardy spaces [5], for each $p$ in $(1, \infty]$ we define an atomic Hardy space $H^{1, p}(\mu)$. Atoms are functions supported in Calderón-Zygmund sets, with vanishing integral and satisfying a certain size condition. We shall prove that all the spaces $H^{1, p}(\mu), p \in(1, \infty]$, coincide and we simply denote by $H^{1}(\mu)$ this atomic Hardy space.

We then find the real interpolation spaces between $H^{1}(\mu)$ and $L^{q}(\mu), q \in(1, \infty]$. The interpolation results which we prove are the analogue of the classical interpolation results (see [7, 11, 13, 14]), but the proofs are different. Indeed, in the classical setting the maximal characterization of the Hardy space is used to obtain the interpolation results, while the Hardy space $H^{1}(\mu)$ introduced in this paper has an atomic definition.

Further, we show that a singular integral operator whose kernel satisfies an integral Hörmander condition, extends to a bounded operator from $H^{1}(\mu)$ to $L^{1}(\mu)$. As a consequence of this result, we show that spectral multipliers of a distinguished Laplacian $\mathscr{L}$ and the first order Riesz transform associated to $\mathscr{L}$ extend to bounded operators from $H^{1}(\mu)$ to $L^{1}(\mu)$.

It would be also interesting to characterize the dual space of $H^{1}(\mu)$ and to obtain complex interpolation results involving $H^{1}(\mu)$, its dual and the $L^{q}(\mu)$-spaces. This will be the object of further investigations.

All the results described above may be considered as an analogue of the classical theory of Hardy spaces.

The classical Hardy space [5, 6, 15] was introduced in $\left(\mathbb{R}^{n}, d, m\right)$, where $d$ is the Euclidean metric and $m$ denotes the Lebesgue measure and more generally on a space of homogeneous type, i.e. a metric measure space $(X, d, \mu)$ where the doubling condition is satisfied, i.e., there exists a constant $C$ such that

$$
\mu(B(x, 2 r)) \leq C \mu(B(x, r)) \quad \forall x \in X, \quad \forall r>0
$$

Extensions of the theory of Hardy spaces have been considered in the literature on various metric measure spaces which do not satisfy the doubling condition (1.3). The literature on this subject is huge and we shall only cite here some contributions [3, 4, 12, 16] which are strictly related to our work.

In particular, we mention that Celotto and Meda [4] studied various Hardy spaces on a homogeneous tree $\mathscr{V}$ endowed with the metric $d$ and the counting measure, which is not the measure $\mu$ that we consider here. Their theory is useful to study the boundedness of singular integral operators related to the standard Laplacian defined on trees which is self-adjoint with respect to the counting measure and not to the measure $\mu$. The theory we develop here instead is useful to study singular integral operators related to a distinguished Laplacian self-adjoint on $L^{2}(\mu)$ (see Subsection 4.3). 
We mention that in [12, 16] the authors used the Calderón-Zygmund theory introduced by Hebisch and Steger in [8] to construct Hardy spaces on some solvable Lie groups of exponential growth and studied their properties. Our work can be thought as a counterpart in a discrete setting of the results in [16], and some of our proofs are strongly inspired by it.

Positive constants are denoted by $C$; these may differ from one line to another, and may depend on any quantifiers written, implicitly or explicitly, before the relevant formula.

\section{Weighted homogeneous tRees}

In this section we introduce the infinite homogeneous tree and we define a distance $d$ and and a measure $\mu$ on it. We show that the corresponding metric measure space $(\mathscr{V}, d, \mu)$ does not satisfy the doubling property. We then introduce a family of sets, called trapezoids, which will be fundamental in the construction of Hardy spaces.

Definition 2.1. An infinite homogeneous tree of order $q+1$ is a graph $T=(\mathscr{V}, \mathscr{E})$, where $\mathscr{V}$ denotes the set of vertices and $\mathscr{E}$ denotes the set of edges, with the following properties:

(i) $T$ is connected and acyclic;

(ii) each vertex has exactly $q+1$ neighbours.

On $\mathscr{V}$ we can define the distance $d(x, y)$ between two vertices $x$ and $y$ as the length of the shortest path between $x$ and $y$. We also fix a doubly-infinite geodesic $g$ in $T$, that is a connected subset $g \subset \mathscr{V}$ such that

(i) for each element $v \in g$ there are exactly two neighbours of $v$ in $g$;

(ii) for every couple $(u, v)$ of elements in $g$, the shortest path joining $u$ and $v$ is contained in $g$.

We define a mapping $N: g \rightarrow \mathbb{Z}$ such that

$$
|N(x)-N(y)|=d(x, y) \quad \forall x, y \in g .
$$

This corresponds to the choice of an origin $o \in g$ (the only vertex for which $N(o)=0$ ) and an orientation for $g$; in this way we obtain a numeration of the vertices in $g$. We define the level function $\ell: \mathscr{V} \rightarrow \mathbb{Z}$ as

$$
\ell(x)=N\left(x^{\prime}\right)-d\left(x, x^{\prime}\right)
$$

where $x^{\prime}$ is the only vertex in $g$ such that $d\left(x, x^{\prime}\right)=\min \{d(x, z): z \in g\}$. For $x, y \in \mathscr{V}$ we say that $y$ lies above $x$ if

$$
\ell(x)=\ell(y)-d(x, y) .
$$

In this case we also say that $x$ lies below $y$.

Let $\mu$ be the measure on $\mathscr{V}$ such that for each function $f: \mathscr{V} \rightarrow \mathbb{C}$

$$
\int_{\mathscr{V}} f d \mu=\sum_{x \in \mathscr{V}} f(x) q^{\ell(x)} .
$$




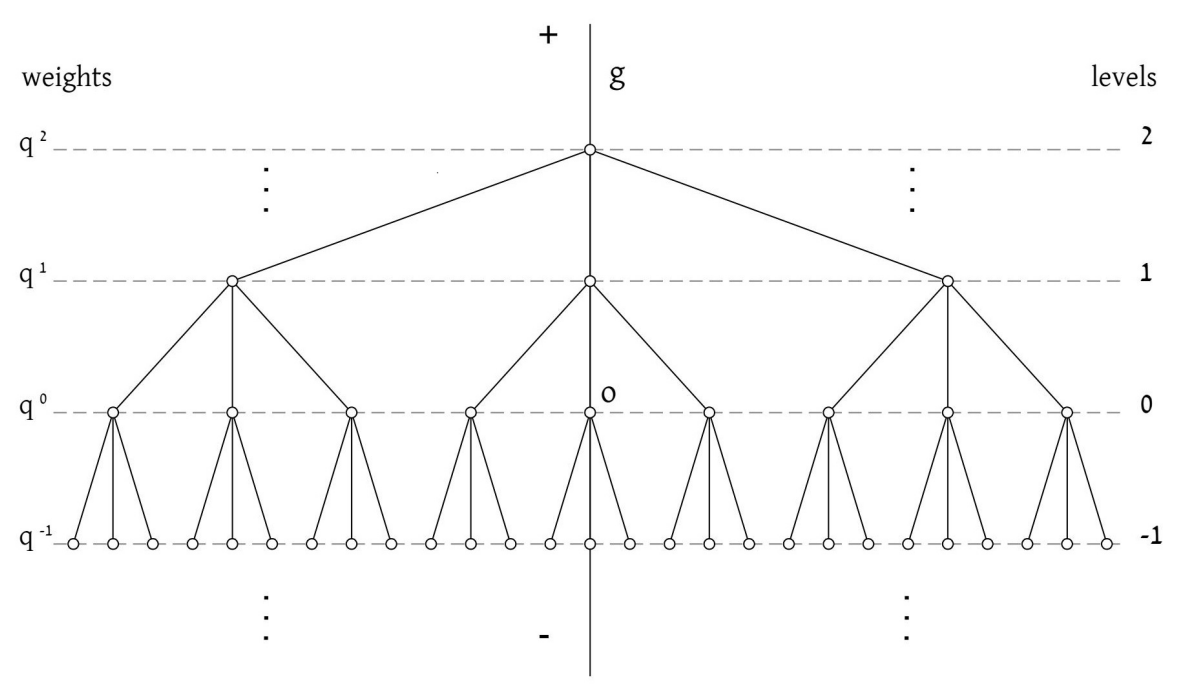

FIGURE 1. Representation of the measure $\mu(q=3)$

Then $\mu$ is a weighted counting measure such that the weight of a vertex depends only on its level and the weight associated to a certain level is given by $q$ times the weight of the level immediately underneath (see Figure 1).

2.1. Doubling and local doubling properties. Observe that the space $(\mathscr{V}, d, \mu)$ exhibits exponential volume growth. Indeed given $x_{0} \in \mathscr{V}$ and $r \geq 1$ consider the sphere $S_{r}\left(x_{0}\right)=$ $\left\{x \in \mathscr{V}: d\left(x, x_{0}\right)=r\right\}$ and the closed ball $B_{r}\left(x_{0}\right)=\left\{x \in \mathscr{V}: d\left(x, x_{0}\right) \leq r\right\}$. A direct computation shows that for $r \geq 1$ their measures are given by:

$$
\mu\left(S_{r}\left(x_{0}\right)\right)=q^{\ell\left(x_{0}\right)+r-1}(1+q) \quad \text { and } \quad \mu\left(B_{r}\left(x_{0}\right)\right)=q^{\ell\left(x_{0}\right)} \frac{q^{r+1}+q^{r}-2}{q-1} .
$$

We notice that they depend on the level of the center $x_{0}$ and grow exponentially with respect to the radius $r$. As a consequence we can prove the following.

Proposition 2.2. The space $(\mathscr{V}, d, \mu)$ is not doubling but it is locally doubling.

Proof. Fix $x_{0} \in \mathscr{V}$ and notice that

$$
\lim _{r \rightarrow \infty} \frac{\mu\left(B_{2 r}\left(x_{0}\right)\right)}{\mu\left(B_{r}\left(x_{0}\right)\right)}=\lim _{r \rightarrow \infty} \frac{q^{\ell\left(x_{0}\right)} \frac{q^{2 r+1}+q^{2 r}-2}{q-1}}{q^{\ell\left(x_{0}\right)} \frac{q^{r+1}+q^{r}-2}{q-1}}=\lim _{r \rightarrow \infty} q^{r}=+\infty .
$$

Thus the doubling property (1.3) fails. 
Instead, we show that $(\mathscr{V}, d, \mu)$ is locally doubling. Indeed, fix $x_{0} \in \mathscr{V}$ and $R>0$ and consider $r \leq R$; one has

$$
\begin{aligned}
\mu\left(B_{2 r}\left(x_{0}\right)\right) & =q^{\ell\left(x_{0}\right)} \frac{q^{2 r+1}+q^{2 r}-2}{q-1} \leq q^{\ell\left(x_{0}\right)} \frac{\frac{q^{2 R+1}+q^{2 R}-2}{q-1}}{\frac{q^{R+1}+q^{R}-2}{q-1}} \frac{q^{r+1}+q^{r}-2}{q-1} \\
& =C_{R} \mu\left(B_{r}\left(x_{0}\right)\right)
\end{aligned}
$$

with $C_{R}=\frac{q^{2 R+1}+q^{2 R}-2}{q^{R+1}+q^{R}-2}>0$ independent of $x_{0}$ and $r$.

2.2. Admissible trapezoids and Calderón-Zygmund sets. In this subsection we introduce the notion of trapezoid and recall the definition and the main properties of the admissible trapezoids introduced in [8].

Definition 2.3. We call trapezoid a set of vertices $S \subset \mathscr{V}$ for which there exist $x_{S} \in \mathscr{V}$ and $a, b \in \mathbb{R}_{+}$such that

$$
S=\left\{x \in \mathscr{V}: x \text { lies below } x_{S}, a \leq \ell\left(x_{S}\right)-\ell(x)<b\right\}
$$

In the following we will refer to $x_{S}$ as the root node of the trapezoid. Among all trapezoids we are mostly interested in those where $a$ and $b$ are related by particular conditions, as specified in the following definitions.

Definition 2.4. A trapezoid $R \subset \mathscr{V}$ is an admissible trapezoid if and only if one of the following occurs:

(i) $R=\left\{x_{R}\right\}$ with $x_{R} \in \mathscr{V}$, that is $R$ consists of a single vertex ;

(ii) $\exists x_{R} \in \mathscr{V}, \exists h \in \mathbb{N}^{+}$such that

$$
R=\left\{x \in \mathscr{V}: x \text { lies below } x_{R}, h \leq \ell\left(x_{R}\right)-\ell(x)<2 h\right\} .
$$

We set $h(R)=1$ in the first case and $h(R)=h$ in the second case. In both cases $h(R)$ can be interpreted as the height of the admissible trapezoid, which coincides with the number of levels spanned by $R$ (see Figure 2 ).

Definition 2.5. We call width of the admissible trapezoid $R$ the quantity

$$
w(R)=q^{\ell\left(x_{R}\right)} .
$$

We have that:

$$
\mu(R)=h(R) q^{\ell\left(x_{R}\right)}=h(R) w(R) .
$$

We now introduce the family of Calderón-Zygmund sets. They are trapezoids, even if not of admissible type; they consist of suitable enlargements of admissible trapezoids, constructed according to the following definition. 


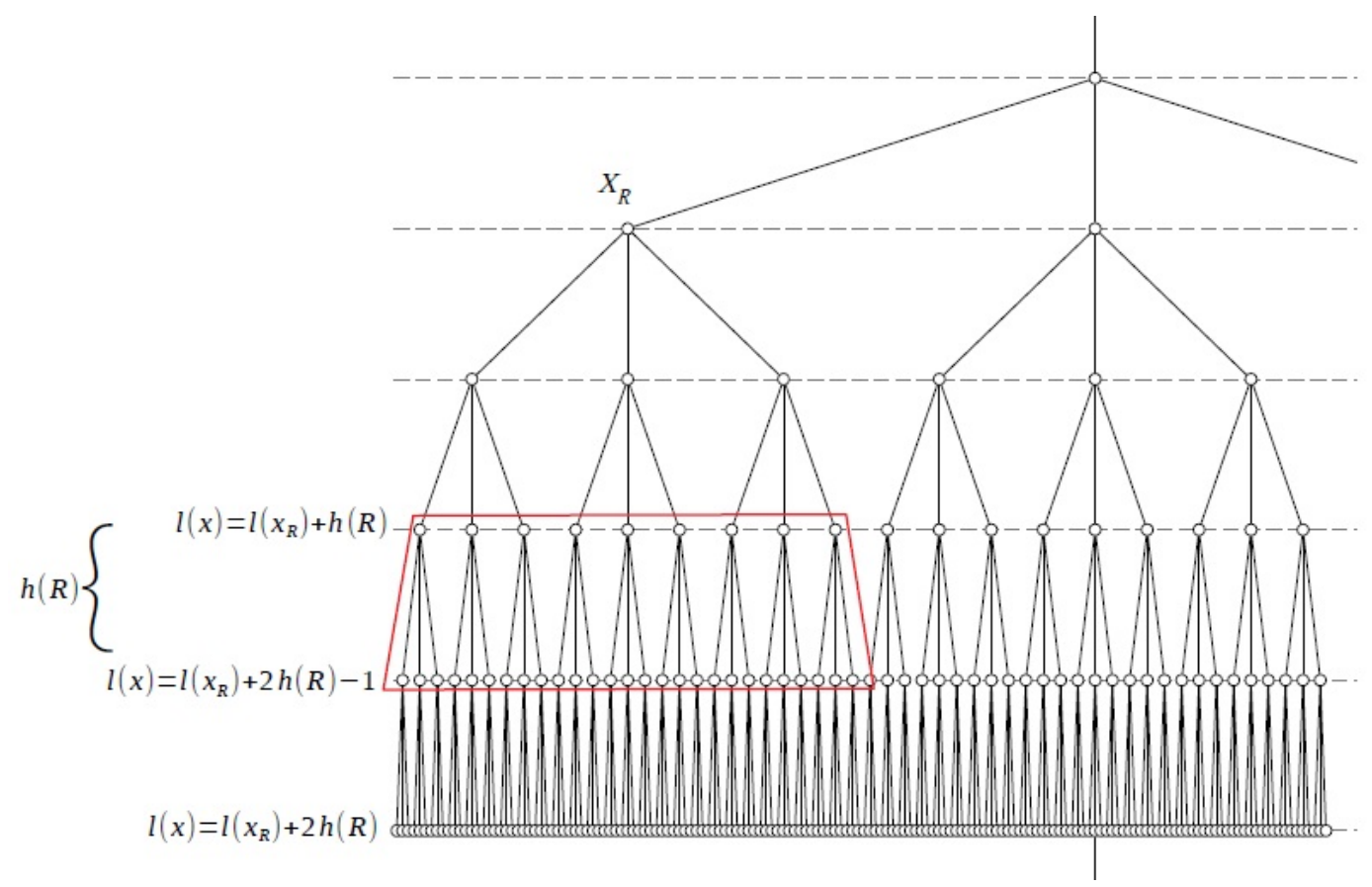

FigURE 2. Representation of an admissible trapezoid with $h(R)=2(q=3)$

Definition 2.6. Given an admissible trapezoid $R$, the envelope of $R$ is the set

$$
\tilde{R}=\left\{x \in \mathscr{V}: x \text { lies below } x_{R}, \frac{h}{2} \leq \ell\left(x_{R}\right)-\ell(x)<4 h\right\}
$$

and we set $h(\tilde{R})=h(R)$. The envelope of an admissible trapezoid is also called a CalderónZygmund set.

Proposition 2.7. Let $R$ be an admissible trapezoid. Then:

$$
\mu(\tilde{R}) \leq 4 \mu(R) .
$$

Proof. In the degenerate case one has $R=\left\{x_{R}\right\}=\tilde{R}$ and then $\mu(\tilde{R})=\mu(R)$. In the nondegenerate case

$$
\begin{aligned}
\mu(\tilde{R}) & =\sum_{\ell=\ell\left(x_{R}\right)-4 h+1}^{\left\lfloor\ell\left(x_{R}\right)-\frac{h}{2}\right\rfloor} \sum_{x \in \tilde{R}: \ell(x)=\ell} q^{\ell}=\sum_{\ell=\ell\left(x_{R}\right)-4 h+1}^{\left\lfloor\ell\left(x_{R}\right)-\frac{h}{2}\right\rfloor} q^{\ell} q^{\ell\left(x_{R}\right)-\ell} \\
& \leq q^{\ell\left(x_{R}\right)}\left(\ell\left(x_{R}\right)-\frac{h}{2}-\ell\left(x_{R}\right)+4 h\right) \\
& \leq 4 \mu(R),
\end{aligned}
$$

which concludes the proof. 
Proposition 2.8. Let $R_{1}$ and $R_{2}$ be two admissible trapezoids. If

$$
R_{1} \cap R_{2} \neq \emptyset \text { and } w\left(R_{1}\right) \geq w\left(R_{2}\right),
$$

then

$$
R_{2} \subset \tilde{R_{1}} \text {. }
$$

Proof. The only nontrivial case is when neither $R_{1}$ nor $R_{2}$ is composed of a single vertex. Let $x_{R_{1}}$ and $x_{R_{2}}$ be the two root nodes of $R_{1}$ and $R_{2}$, respectively. Then

$$
w\left(R_{1}\right)=q^{\ell\left(x_{R_{1}}\right)} \geq q^{\ell\left(x_{R_{2}}\right)}=w\left(R_{2}\right) \quad \Longrightarrow \quad \ell\left(x_{R_{1}}\right) \geq \ell\left(x_{R_{2}}\right) .
$$

Moreover, since $R_{1} \cap R_{2} \neq \emptyset, x_{R_{2}}$ is below $x_{R_{1}}$ and so is every vertex of $R_{2}$. In the following we denote $h_{1}=h\left(R_{1}\right)$ and $h_{2}=h\left(R_{2}\right)$. Let $\hat{x} \in R_{1} \cap R_{2} \neq \emptyset$. Then we obtain the following constraints:

$$
\left\{\begin{array} { l } 
{ \ell ( x _ { R _ { 2 } } ) - 2 h _ { 2 } + 1 \leq \ell ( \hat { x } ) \leq \ell ( x _ { R _ { 1 } } ) - h _ { 1 } } \\
{ \ell ( x _ { R _ { 1 } } ) - 2 h _ { 1 } + 1 \leq \ell ( \hat { x } ) \leq \ell ( x _ { R _ { 2 } } ) - h _ { 2 } }
\end{array} \Longrightarrow \left\{\begin{array}{l}
\ell\left(x_{R_{1}}\right)-\ell\left(x_{R_{2}}\right) \geq h_{1}-2 h_{2}+1 \\
\ell\left(x_{R_{1}}\right)-\ell\left(x_{R_{2}}\right) \leq 2 h_{1}-h_{2}-1 .
\end{array}\right.\right.
$$

Let $x \in R_{2}$; then $x$ lies below $x_{R_{1}}$. Moreover

$$
\begin{aligned}
\ell\left(x_{R_{1}}\right)-\ell(x) & =\left[\ell\left(x_{R_{1}}\right)-\ell\left(x_{R_{2}}\right)\right]+\left[\ell\left(x_{R_{2}}\right)-\ell(x)\right] \\
& \leq 2\left[2 h_{1}-h_{2}-1\right]+\left[2 h_{2}-1\right]<4 h_{1} . \\
\ell\left(x_{R_{1}}\right)-\ell(x) & =\left[\ell\left(x_{R_{1}}\right)-\ell\left(x_{R_{2}}\right)\right]+\left[\ell\left(x_{R_{2}}\right)-\ell(x)\right] \\
& \geq \frac{1}{2}\left[h_{1}-2 h_{2}+1\right]+\left[h_{2}\right]>\frac{h_{1}}{2} .
\end{aligned}
$$

So $\frac{h_{1}}{2} \leq \ell\left(x_{R_{1}}\right)-\ell(x)<4 h_{1}, \forall x \in R_{2}$ and this shows that $R_{2} \subset \tilde{R_{1}}$.

Proposition 2.9. Given a Calderón-Zygmund set $\tilde{R}$, we have that for all $z \in \tilde{R}$

$$
\tilde{R} \subset B(z, 8 h(\tilde{R})) .
$$

Proof. Fix a point $z \in \tilde{R}$. Every vertex $y \in \tilde{R}$ has distance $d(z, y) \leq 8 h(\tilde{R})-2<8 h(\tilde{R})$. Indeed, starting from $z$ it is possible to reach $y$ passing through at most $[(4 h(\tilde{R})-1)] 2=8 h(\tilde{R})-2$ edges, moving from $z$ to the root node of the trapezoid and then from the root node to $y$.

Definition 2.10. Given a Calderón-Zygmund set $\tilde{R}$, we define the set

$$
\tilde{R}^{*}=\{x \in \mathscr{V}: d(x, \tilde{R})<h(\tilde{R}) / 4\} .
$$

It is easy to see that there exists a positive constant $C$ such that for every Calderón-Zygmund set $\tilde{R}$

$$
\mu\left(\tilde{R}^{*}\right) \leq C \mu(\tilde{R})
$$

See [1, p.75] for a proof of this fact. 


\section{The MAXIMAL FUnCtion}

In this section we define two maximal functions and describe a way to construct a covering of their level sets which will be useful in the sequel.

Definition 3.1. Given $f: \mathscr{V} \rightarrow \mathbb{C}$, we define the maximal function $M$ as

$$
M f(x)=\sup _{R: x \in R} \frac{1}{\mu(R)} \int_{R}|f| d \mu
$$

where the supremum is taken over all admissible trapezoids $R$ containing $x$.

Consider a function $f \in L^{p}(\mu)$ and let $\lambda>0$. We are interested in constructing a covering of the level set

$$
\Omega_{\lambda^{p}}=\left\{x \in \mathscr{V}: M\left(|f|^{p}\right)(x)>\lambda^{p}\right\} .
$$

Define $S_{0}$ as the family of all admissible trapezoids $R$ such that

$$
\int_{R}|f|^{p} d \mu \geq \lambda^{p} \mu(R)
$$

Since $S_{0}$ is countable, we can introduce an ordering in $S_{0}$. All trapezoids in $S_{0}$ have bounded measure and bounded width, because $\forall R \in S_{0}$ we have

$$
w(R)=\frac{\mu(R)}{h(R)} \leq \mu(R) \leq \frac{1}{\lambda^{p}}\|f\|_{L^{p}}^{p} .
$$

So it is possible to choose in $S_{0}$ a trapezoid $R_{0}$ of largest width (in case of ties, we choose that trapezoid of largest width which occurs earliest in the ordering). Then we proceed inductively:

(i) $S_{i+1}$ is the family of all admissible trapezoids $R \in S_{i}$ disjoint from $R_{0}, \ldots, R_{i}$;

(ii) $R_{i+1}$ is the trapezoid of largest width in $S_{i+1}$ which occurs earliest in the ordering.

Let $R \in S_{0}$. Then by construction $R$ intersects some $R_{i}$ with $w\left(R_{i}\right) \geq w(R)$.

Indeed, there exists a number $j \in\{0,1,2, \ldots\}$ such that $R \in S_{j}$ and $R \notin S_{j+1}$, i.e. in the previous construction there exists a step $j$ in which one of the following occurs:

(1) either $R$ is the trapezoid of largest width that occurs earliest in the ordering, and then $R$ is selected and $R_{j}=R$, so that $R \cap R_{i} \neq \emptyset$ for $i=j$;

(2) or $R$ is not the trapezoid of largest width that occurs earliest in the ordering and it intersects $R_{j}$. Then $R$ is not in $S_{i} \forall i \geq j+1$ and $R \cap R_{i} \neq \emptyset$ for $i=j$.

To ensure that there is some $j$ with the stated property it is sufficient to avoid that $S_{0}$ can contain an infinite number of trapezoids with the same width that do not intersect each other. This possibility is excluded observing that:

$$
\sum_{i} \mu\left(R_{i}\right) \leq \frac{1}{\lambda^{p}} \sum_{i} \int_{R_{i}}|f|^{p} d \mu \leq \frac{1}{\lambda^{p}} \int_{\mathscr{V}}|f|^{p} d \mu=\frac{1}{\lambda^{p}}\|f\|_{L^{p}}<\infty,
$$


while if there was among the $R_{i}$ 's an infinite number of trapezoids with constant width $w$ we would have

$$
\sum_{i} \mu\left(R_{i}\right) \geq \sum_{n=1}^{\infty} w=\infty
$$

In conclusion,

$$
\forall R \in S_{0}, \quad \exists i: R \cap R_{i} \neq \emptyset \text { and } w\left(R_{i}\right) \geq w(R) .
$$

By Proposition 2.8, this implies $R \subset \tilde{R}_{i}$. We set $E:=\bigcup_{i} \tilde{R}_{i}$. We have that $\Omega_{\lambda^{p}} \subset E$ and

$$
\mu(E) \leq \sum_{i} \mu\left(\tilde{R}_{i}\right) \leq 4 \sum_{i} \mu\left(R_{i}\right) \leq \frac{4\|f\|_{L^{p}}}{\lambda^{p}} .
$$

Definition 3.2. Given $f: \mathscr{V} \rightarrow \mathbb{C}$ and a Calderón-Zygmund set $\tilde{Q}$, we define the maximal function $M_{\tilde{Q}}$ as follows

$$
M_{\tilde{Q}}(f)(x)=\sup _{R \subset \tilde{Q}: x \in R} \mu(R)^{-1} \int_{R}|f| d \mu \quad \forall x \in \tilde{Q},
$$

where the supremum is taken over all admissible trapezoids $R$ containing $x$ and contained in $\tilde{Q}$. When $x \notin \tilde{Q}$ we set $M_{\tilde{Q}}(f)(x)=0$.

Consider a function $f \in L^{p}(\mu)$ with support contained in a Calderón-Zygmund set $\tilde{Q}$, and let $\lambda>0$. We define

$$
\Omega_{\tilde{Q}, \lambda^{p}}=\left\{x \in \mathscr{V}: M_{\tilde{Q}}\left(|f|^{p}\right)(x)>\lambda^{p}\right\} .
$$

Arguing as before, we can show that there exists a family of pairwise disjoint admissible trapezoids $\left\{R_{i}\right\}$ such that $R_{i} \subset \tilde{Q}, \sum_{i} \mu\left(R_{i}\right) \leq \frac{1}{\lambda^{p}}\|f\|_{L^{p}}$ and $\Omega_{\tilde{Q}, \lambda^{p}} \subset \bigcup_{i} \tilde{R}_{i}$.

\section{Hardy SPACES}

In this section we define atomic Hardy spaces replacing balls with Calderón-Zygmund sets in the classical definition of atoms.

Definition 4.1. A function $a$ is a $(1, p)$-atom, for $p \in(1, \infty]$, if it satisfies the following properties:

(i) $a$ is supported in a Calderón-Zygmund set $\tilde{R}$;

(ii) $\|a\|_{L^{p}} \leq \mu(\tilde{R})^{1 / p-1}$;

(iii) $\int_{\mathscr{V}} a \mathrm{~d} \mu=0$.

Observe that a $(1, p)$-atom is in $L^{1}(\mu)$ and it is normalized in such a way that its $L^{1}$-norm does not exceed 1.

Definition 4.2. The Hardy space $H^{1, p}(\mu)$ is the space of all functions $h$ in $L^{1}(\mu)$ such that $h=\sum_{j} \lambda_{j} a_{j}$, where $a_{j}$ are $(1, p)$-atoms and $\lambda_{j}$ are complex numbers such that $\sum_{j}\left|\lambda_{j}\right|<\infty$. We denote by $\|h\|_{H^{1, p}}$ the infimum of $\sum_{j}\left|\lambda_{j}\right|$ over all decompositions $h=\sum_{j} \lambda_{j} a_{j}$, where $a_{j}$ are $(1, p)$-atoms.

The space $H^{1, p}(\mu)$ endowed with the norm $\|\cdot\|_{H^{1, p}}$ is a Banach space. 
4.1. Equivalence of spaces $H^{1, p}(\mu)$ for $p \in(1, \infty]$. It easily follows from the above definitions that $H^{1, \infty}(\mu) \subseteq H^{1, p}(\mu)$, whenever $p \in(1, \infty)$. Actually we shall prove that $H^{1, \infty}(\mu)=H^{1, p}(\mu)$, for every $p \in(1, \infty)$. To show this fact we first prove a preliminary result.

Proposition 4.3. Let a be a $(1, p)$-atom, where $p \in(1, \infty)$. Then a is in $H^{1, \infty}(\mu)$ and there exists a positive constant $C_{p}$, which depends only on $p$, such that

$$
\|a\|_{H^{1, \infty}} \leq C_{p} .
$$

Proof. Let $a$ be a $(1, p)$-atom supported in a Calderón-Zygmund set $\tilde{Q}$. We define $b:=\mu(\tilde{Q}) a$.

Let $\alpha$ be a positive number such that $\alpha>2\left[24 q\left(1+4^{p}\right)\right]^{1 /(p-1)}$.

We shall prove that for all $n \in \mathbb{N}$ there exist functions $a_{j_{\ell}}, h_{j_{n}}$ and admissible sets $\tilde{R}_{j_{\ell}}, j_{\ell} \in \mathbb{N}^{\ell}$, $\ell=0, \ldots, n$, such that

$$
b=\sum_{\ell=0}^{n-1} 4(6 q)^{1 / p} \alpha^{\ell+1} \sum_{j_{\ell}} \mu\left(\tilde{R}_{j_{\ell}}\right) a_{j_{\ell}}+\sum_{j_{n} \in \mathbb{N}^{n}} f_{j_{n}},
$$

where the following properties are satisfied:

(i) $a_{j \ell}$ is a $(1, \infty)$-atom supported in the Calderón-Zygmund set $\tilde{R}_{j_{\ell}}$;

(ii) $f_{j_{n}}$ is supported in $R_{j_{n}}$ and $\int f_{j_{n}} \mathrm{~d} \mu=0$;

(iii) $\left(\frac{1}{\mu\left(\tilde{R}_{j_{n}}\right)} \int_{\tilde{R}_{j_{n}}}\left|f_{j_{n}}\right|^{p} \mathrm{~d} \mu\right)^{1 / p} \leq 2^{1-1 / p}(6 q)^{1 / p}\left(1+4^{p}\right)^{1 / p} \alpha^{n}$;

(iv) $\left|f_{j_{n}}(x)\right| \leq|b(x)|+4(6 q)^{1 / p} n \alpha^{n}, \quad \forall x \in \tilde{R}_{j_{n}}$;

(v) $\sum_{j_{n}} \mu\left(\tilde{R}_{j_{n}}\right) \leq 4^{n+1}\left[2^{p-1}(6 q)\left(1+4^{p}\right)\right]^{n} \alpha^{-n p}\|b\|_{L^{p}}^{p}$.

We first suppose that the decomposition 4.1 exists and we show that $a \in H^{1, \infty}(\mu)$. Set $F_{n}=\sum_{j_{n}} f_{j_{n}}$. We prove that $F_{n} \in L^{1}(\mu)$ and that its $L^{1}$-norm tends to zero when $n$ tends to $\infty$. Indeed, by Hölder's inequality

$$
\left\|F_{n}\right\|_{L^{1}} \leq \sum_{j_{n} \in \mathbb{N}^{n}}\left\|f_{j_{n}}\right\|_{L^{1}} \leq \sum_{j_{n} \in \mathbb{N}^{n}} \mu\left(\tilde{R}_{j_{n}}\right)^{1 / p^{\prime}}\left\|f_{j_{n}}\right\|_{L^{p}}
$$

where $p^{\prime}$ is the conjugate exponent of $p$. Now by (iii) and (v) we have that

$$
\begin{aligned}
\left\|F_{n}\right\|_{L^{1}} & \leq \sum_{j_{n} \in \mathbb{N}^{n}} \mu\left(\tilde{R}_{j_{n}}\right)^{1 / p^{\prime}} \mu\left(\tilde{R}_{j_{n}}\right)^{1 / p} 2^{1-1 / p}(6 q)^{1 / p}\left(1+4^{p}\right)^{1 / p} \alpha^{n} \\
& \leq 4^{n+1}\left[2^{p-1} 6 q\left(1+4^{p}\right)\right]^{n} \alpha^{-n p}\|b\|_{L^{p}}^{p} 2^{1-1 / p}(6 q)^{1 / p}\left(1+4^{p}\right)^{1 / p} \alpha^{n},
\end{aligned}
$$

which tends to zero when $n$ tends to $\infty$, since $\alpha>2\left[24 q\left(1+4^{p}\right)\right]^{1 /(p-1)}$.

This shows that the series $\sum_{\ell=0}^{n-1} 4(6 q)^{1 / p} \alpha^{\ell+1} \sum_{j_{\ell}} \mu\left(\tilde{R}_{j_{\ell}}\right) a_{j \ell}$ converges to $b$ in $L^{1}(\mu)$. Moreover by (v) we deduce that

$$
\begin{aligned}
\sum_{\ell=0}^{\infty} 4(6 q)^{1 / p} \alpha^{\ell+1} \sum_{j_{\ell}} \mu\left(\tilde{R}_{j \ell}\right) & \leq \sum_{\ell=0}^{\infty} 4(6 q)^{1 / p} \alpha^{\ell+1} 4^{\ell+1}\left[2^{p-1}(6 q)\left(1+4^{p}\right)\right]^{\ell} \alpha^{-\ell p}\|b\|_{L^{p}}^{p} \\
& \leq C_{p}\|b\|_{L^{p}}^{p} \leq C_{p} \mu(\tilde{Q}),
\end{aligned}
$$

where $C_{p}$ depends only on $p$. 
It follows that $b$ is in $H^{1, \infty}(\mu)$ and $\|b\|_{H^{1, \infty}} \leq C_{p} \mu(\tilde{Q})$. Thus $a=\mu(\tilde{Q})^{-1} b$ is in $H^{1, \infty}(\mu)$ and $\|a\|_{H^{1, \infty}} \leq C_{p}$, as required.

It remains to prove that the decomposition 4.1) exists. We prove it by induction on $n$.

Step $n=1$. Define

$$
\Omega_{\tilde{Q}, \alpha^{p}}=\left\{x \in \mathscr{V}: M_{\tilde{Q}}\left(|b|^{p}\right)(x)>\alpha^{p}\right\} .
$$

If $\Omega=\emptyset$, then

$$
\mu\left(\left\{x_{R}\right\}\right)^{-1}\left|b\left(x_{R}\right)\right|^{p} \mu\left(\left\{x_{R}\right\}\right) \leq \alpha^{p}, \quad \forall x_{R} \in \mathscr{V} .
$$

It follows that $\|b\|_{L^{\infty}} \leq \alpha$ and we have $b=\alpha \mu(\tilde{Q}) a_{j_{0}}$, so that and (4.1) is satisfied with the $(1, \infty)$-atom $a_{j_{0}}=\alpha^{-1} \mu(\tilde{Q})^{-1} b$ and $f_{j_{1}}=0$ for every $j_{1} \in \mathbb{N}$.

If $\Omega \neq \emptyset$, then we construct a family of trapezoids $R_{i}, i \in \mathbb{N}$, and the corresponding CalderónZygmund sets $\tilde{R}_{i}, i \in \mathbb{N}$, as in Section 3 We then define $U_{i}=\tilde{R}_{i} \backslash\left(\cup_{j<i} \tilde{R}_{j}\right)$, and $h_{i}=b \chi_{U_{i}}$. One can show as in [8, p.43]

$$
\int_{U_{i}}\left|h_{i}\right|^{p} d \mu \leq 6 q \alpha^{p} \mu\left(\tilde{R}_{i}\right) .
$$

See also [1, p.74] for a detailed proof of the previous inequality.

We now define

$$
f_{i}=h_{i}-\mu\left(R_{i}\right)^{-1} \int h_{i} d \mu \chi_{R_{i}}, \quad g=b-\sum_{i} f_{i}
$$

Notice that $f_{i}$ is supported in $\tilde{Q} \cap \tilde{R}_{i}$ and $g$ is supported in $\tilde{Q}$. The average of $f_{i}$ vanishes by construction. Moreover, for every $x \in \tilde{R}_{i}$ we have that

$$
\begin{aligned}
\left|f_{i}(x)\right| & \leq\left|h_{i}(x)\right|+\mu\left(R_{i}\right)^{-1} \int\left|h_{i}\right| d \mu \chi_{R_{i}} \\
& \leq\left|h_{i}(x)\right|+\mu\left(R_{i}\right)^{-1}\left(\int\left|h_{i}\right|^{p} d \mu\right)^{1 / p} \mu\left(\tilde{R}_{i}\right)^{1 / p^{\prime}} \\
& \leq|b(x)|+(6 q)^{1 / p} 4 \alpha,
\end{aligned}
$$

where we have applied (4.2) and Proposition 2.7. It follows that

$$
\left\|f_{i}\right\|_{L^{p}}^{p} \leq 2^{p-1}\left\|h_{i}\right\|_{L^{p}}^{p}+6 q 4^{p} \alpha^{p} \mu\left(\tilde{R}_{i}\right) \leq 2^{p-1} 6 q \alpha^{p}\left(1+4^{p}\right) \mu\left(\tilde{R}_{i}\right) .
$$

Moreover,

$$
\sum_{i} \mu\left(\tilde{R}_{i}\right) \leq 4 \sum_{i} \mu\left(R_{i}\right) \leq 4 \frac{1}{\alpha^{p}} \sum_{i} \int_{R_{i}}|b(x)|^{p} d \mu(x) \leq 4 \frac{1}{\alpha^{p}}\|b\|_{L^{p}}^{p} .
$$

This implies that

$$
\sum_{i}\left\|f_{i}\right\|_{L^{p}}^{p} \leq 2^{p-1} 6 q \alpha^{p}\left(1+4^{p}\right) 4 \frac{1}{\alpha^{p}}\|b\|_{L^{p}}^{p} .
$$

We now estimate the function $g$. If $x$ is a vertex in the complement of $\tilde{Q}$, then obviously $g(x)=0$. If $x$ is a vertex in $\tilde{Q} \cap\left(\bigcup_{i} \tilde{R}_{i}\right)^{c}$, then $g(x)=b(x)$ and $M_{\tilde{Q}}\left(|b|^{p}\right)(x) \leq \alpha^{p}$. Thus $|g(x)|=|b(x)| \leq \alpha$. Let us now take $x \in \tilde{Q} \cap\left(\bigcup_{i} \tilde{R}_{i}\right)$ and let $i_{0}$ be the unique index such that $x \in U_{i_{0}}$. We distinguish two different cases. If $x \notin \bigcup_{i} R_{i}$, then $f_{i_{0}}(x)=h_{i_{0}}(x)=b(x)$ and $f_{i}(x)=0$ for every $i \neq i_{0}$, so 
that $g(x)=b(x)-b(x)=0$. If $x \in \bigcup_{i} R_{i}$, let $i_{1}$ be the unique index such that $x \in R_{i_{1}}$. When $i_{1}=i_{0}$ we have that $f_{i}(x)=0$ for every $i \neq i_{0}$ and

$$
g(x)=b(x)-f_{i_{0}}(x)=b(x)-b(x)+\mu\left(R_{i_{0}}\right)^{-1} \int h_{i_{0}} d \mu,
$$

so that $|g(x)| \leq(6 q)^{1 / p} 4 \alpha$. When $i_{1} \neq i_{0}$ we have that $f_{i}(x)=0$ for every $i \neq i_{0}, i_{1}$ and

$$
g(x)=b(x)-f_{i_{0}}(x)-f_{i_{1}}(x)=b(x)-b(x)+\mu\left(R_{i_{1}}\right)^{-1} \int h_{i_{1}} d \mu,
$$

so that $|g(x)| \leq(6 q)^{1 / p} 4 \alpha$.

In conclusion,

$$
b(x)=(6 q)^{1 / p} 4 \alpha \mu(\tilde{Q}) a(x)+\sum_{j_{1} \in \mathbb{N}} f_{j_{1}}(x),
$$

where $a=(6 q)^{-1 / p} 4^{-1} \alpha^{-1} \mu(\tilde{Q})^{-1} g$ is a $(1, \infty)$-atom supported in $\tilde{Q}$ and all properties (i)-(v) are satisfied.

Inductive step. Suppose that a decomposition

$$
b=\sum_{\ell=0}^{n-1} 4(6 q)^{1 / p} \alpha^{\ell+1} \sum_{j_{\ell}} \mu\left(\tilde{R}_{j_{\ell}}\right) a_{j_{\ell}}+\sum_{j_{n} \in \mathbb{N}^{n}} f_{j_{n}},
$$

holds, where properties (i)-(v) are satisfied. We shall prove that a similar decomposition of $b$ holds with $n+1$ in place of $n$. To do so, we decompose each function $f_{j_{n}}$ by following the same construction applied above to $b$ with respect to $\alpha^{n+1}$. We omit the details.

The following theorem is now an easy consequence of Proposition 4.3

Theorem 4.4. For any $p \in(1, \infty), H^{1, p}(\mu)=H^{1, \infty}(\mu)$ and the norms $\|\cdot\|_{H^{1, p}}$ and $\|\cdot\|_{H^{1, \infty}}$ are equivalent.

In the sequel we denote by $H^{1}(\mu)$ the space $H^{1, \infty}(\mu)$ and we define $\|\cdot\|_{H^{1}}=\|\cdot\|_{H^{1, \infty}}$.

4.2. Real interpolation properties of $H^{1}(\mu)$. In this subsection we study the real interpolation of $H^{1}(\mu)$ and the $L^{p}(\mu)$ spaces. We first recall some notation of the real interpolation of normed spaces, focusing on the $K$-method. For the details see [2].

Given two compatible normed spaces $A_{0}$ and $A_{1}$, for any $t>0$ and for any $a \in A_{0}+A_{1}$ we define

$$
K\left(t, a ; A_{0}, A_{1}\right)=\inf \left\{\left\|a_{0}\right\|_{A_{0}}+t\left\|a_{1}\right\|_{A_{1}}: a=a_{0}+a_{1}, a_{i} \in A_{i}\right\} .
$$

Take $q \in[1, \infty]$ and $\theta \in(0,1)$. The real interpolation space $\left[A_{0}, A_{1}\right]_{\theta, q}$ is defined as the set of the elements $a \in A_{0}+A_{1}$ such that

$$
\|a\|_{\theta, q}= \begin{cases}\left(\int_{0}^{\infty}\left[t^{-\theta} K\left(t, a ; A_{0}, A_{1}\right)\right]^{q} \frac{\mathrm{d} t}{t}\right)^{1 / q} & \text { if } 1 \leq q<\infty \\ \left\|t^{-\theta} K\left(t, a ; A_{0}, A_{1}\right)\right\|_{\infty} & \text { if } q=\infty,\end{cases}
$$

is finite. The space $\left[A_{0}, A_{1}\right]_{\theta, q}$ endowed with the norm $\|a\|_{\theta, q}$ is an exact interpolation space of exponent $\theta$. 
We refer the reader to [10] for an overview of the real interpolation results which hold in the classical setting. Our aim is to prove the same results in our context. Note that in our case a maximal characterization of $H^{1}(\mu)$ is not avalaible, so that we cannot follow the classical proofs but we shall only use the atomic definition of $H^{1}(\mu)$ to prove the results.

We shall first estimate the $K$ functional of $L^{p}$-functions with respect to the couple of spaces $\left(H^{1}(\mu), L^{p_{1}}(\mu)\right), 1<p_{1} \leq \infty$.

Lemma 4.5. Suppose that $1<p<p_{1} \leq \infty$ and let $\theta \in(0,1)$ be such that $\frac{1}{p}=1-\theta+\frac{\theta}{p_{1}}$. Let $f$ be in $L^{p}(\mu)$. The following hold:

(i) for every $\lambda>0$ there exists a decomposition $f=g^{\lambda}+b^{\lambda}$ in $L^{p_{1}}(\mu)+H^{1}(\mu)$ such that

(i') $\left\|g^{\lambda}\right\|_{L^{\infty}} \leq C \lambda$ and, if $p_{1}<\infty$, then $\left\|g^{\lambda}\right\|_{L_{1}^{p}}^{p_{1}} \leq C \lambda^{p_{1}-p}\|f\|_{L^{p}}^{p}$;

(i”) $\left\|b^{\lambda}\right\|_{H^{1}} \leq C \lambda^{1-p}\|f\|_{L^{p}}^{p}$;

(ii) for any $t>0, K\left(t, f ; H^{1}(\mu), L^{p_{1}}(\mu)\right) \leq C t^{\theta}\|f\|_{L^{p}}$;

(iii) $f \in\left[H^{1}(\mu), L^{p_{1}}(\mu)\right]_{\theta, \infty}$ and $\|f\|_{\theta, \infty} \leq C\|f\|_{L^{p}}$.

Proof. Let $f$ be in $L^{p}(\mu)$. We first prove (i). Given a positive $\lambda$, let

$$
\Omega_{\lambda^{p}}=\left\{x \in \mathscr{V}: M\left(|f|^{p}\right)(x)>\lambda^{p}\right\} .
$$

Let $\left\{R_{i}\right\}$ be the collection of trapezoids constructed as in Section 3 We now define $U_{i}=$ $\tilde{R}_{i} \backslash\left(\cup_{j<i} \tilde{R}_{j}\right)$ and $h_{i}=f \chi_{U_{i}}$,

$$
f_{i}=f-\mu\left(R_{i}\right)^{-1} \int h_{i} d \mu \chi_{R_{i}}, \quad b^{\lambda}=\sum_{i} f_{i} \quad \text { and } \quad g^{\lambda}=f-b^{\lambda} .
$$

Arguing as we did in the proof of Proposition 4.3 we can show that

$$
\left\|g^{\lambda}\right\|_{L^{\infty}} \leq C \lambda \quad \text { and } \quad \frac{1}{\mu\left(R_{i}\right)} \int_{R_{i}}\left|h_{i}\right|^{p} d \mu \leq C \lambda^{p} .
$$

If $p_{1}<\infty$, then

$$
\left\|g^{\lambda}\right\|_{L^{p_{1}}}^{p_{1}} \leq \int_{\left(\cup \tilde{R}_{i}\right)^{c}}|f|^{p_{1}} d \mu+\sum_{i} \int_{\tilde{R}_{i}}\left|f-\sum_{j} f_{j}\right|^{p_{1}} d \mu=I+I I .
$$

To estimate $I$ we notice that $\left(\cup_{i} \tilde{R}_{i}\right)^{c} \subset \Omega_{\lambda^{p}}^{c}$, so that

$$
I \leq \int_{\left(\cup \tilde{R}_{i}\right)^{c}}|f|^{p_{1}-p}|f|^{p} d \mu \leq C \lambda^{p_{1}-p}\|f\|_{L^{p}}^{p}
$$

To estimate $I I$ we first observe that given $i$ and $x \in \tilde{R}_{i}$ there exists only two indeces $i_{0} \leq i$ and $i_{1}$ such that $x \in U_{i_{0}}$ and $x \in R_{i_{1}}$. If $i_{0}=i_{1}$, then

$$
f(x)-\sum_{j} f_{j}(x)=f(x)-f_{i_{0}}(x)=\mu\left(R_{i_{0}}\right)^{-1} \int h_{i_{0}} d \mu,
$$

so that $\left|f(x)-\sum_{j} f_{j}(x)\right| \leq C \lambda$. If $i_{0} \neq i_{1}$, then

$$
f(x)-\sum_{j} f_{j}(x)=f(x)-f_{i_{0}}(x)-f_{i_{1}}(x)=\mu\left(R_{i_{1}}\right)^{-1} \int h_{i_{1}} d \mu,
$$


so that $\left|f(x)-\sum_{j} f_{j}(x)\right| \leq C \lambda$. It follows that

$$
I I \leq C \sum_{i} \int_{\tilde{R}_{i}} \lambda^{p_{1}} d \mu \leq C \lambda^{p_{1}} \sum_{i} \mu\left(\tilde{R}_{i}\right) \leq C \lambda^{p_{1}-p}\|f\|_{L^{p}}^{p} .
$$

In conclusion, $\left\|g^{\lambda}\right\|_{L^{p_{1}}}^{p_{1}} \leq C \lambda^{p_{1}-p}\|f\|_{L^{p}}^{p}$.

We now prove that $b^{\lambda}$ is in $H^{1, p}(\mu)$. Indeed, for any $i, f_{i}$ is supported in $\tilde{R}_{i}$, has vanishing integral and

$$
\left\|f_{i}\right\|_{L^{p}} \leq C\left\|h_{i}\right\|_{L^{p}}+C \mu\left(R_{i}\right)^{-1} \mu\left(\tilde{R}_{i}\right)^{1 / p} \int\left|h_{i}\right| d \mu \mu\left(\tilde{R}_{i}\right)^{1 / p} \leq C \lambda .
$$

This shows that $f_{i} \in H^{1, p}(\mu)=H^{1}(\mu)$ and $\left\|f_{i}\right\|_{H^{1}} \leq C \lambda \mu\left(\tilde{R}_{i}\right)$. Since $b^{\lambda}=\sum_{i} f_{i}, b^{\lambda}$ is in $H^{1}(\mu)$ and

$$
\left\|b^{\lambda}\right\|_{H^{1}} \leq C \lambda \sum_{i} \mu\left(\tilde{R}_{i}\right) \leq C \lambda \frac{\|f\|_{L^{p}}^{p}}{\lambda^{p}},
$$

as required.

We now prove (ii). Fix $t>0$. For any positive $\lambda$, let $f=g^{\lambda}+b^{\lambda}$ be the decomposition of $f$ in $L^{p_{1}}(\mu)+H^{1}(\mu)$ given by (i). Thus

$$
\begin{aligned}
K\left(t, f ; H^{1}(\mu), L^{p_{1}}(\mu)\right) & \leq \inf _{\lambda>0}\left(\left\|b^{\lambda}\right\|_{H^{1}}+t\left\|g^{\lambda}\right\|_{L^{p_{1}}}\right) \\
& \leq C \inf _{\lambda>0}\left(\lambda^{1-p}\|f\|_{L^{p}}^{p}+t \lambda^{1-p / p_{1}}\|f\|_{L^{p}}^{p / p_{1}}\right) \\
& =C\|f\|_{L^{p}}^{p / p_{1}} \inf _{\lambda>0} G(t, \lambda),
\end{aligned}
$$

where $G(t, \lambda)=\lambda^{1-p}\|f\|_{L^{p}}^{p\left(1-1 / p_{1}\right)}+t \lambda^{1-p / p_{1}}$. Since

$$
\partial_{\lambda} G(t, \lambda)=\lambda^{-p}\left[(1-p)\|f\|_{L^{p}}^{p\left(1-1 / p_{1}\right)}+\left(1-p / p_{1}\right) t \lambda^{-p / p_{1}+p}\right],
$$

we have that if $p_{1}<\infty$, then

$$
\inf _{\lambda>0} G(t, \lambda)=G\left(t, C_{p}\|f\|_{L^{p}} t^{p_{1} / p-p p_{1}}\right)=C_{p}\|f\|_{L^{p}}^{1-p / p_{1}} t^{\frac{p_{1}(p-1)}{p\left(p_{1}-1\right)}} .
$$

If $p_{1}=\infty$, then

$$
\inf _{\lambda>0} G(t, \lambda)=G\left(t, C_{p}\|f\|_{L^{p}} t^{-1 / p}\right)=C_{p}\|f\|_{L^{p}} t^{1-1 / p}
$$

It follows that

$$
K\left(t, f ; H^{1}, L^{p_{1}}\right) \leq C_{p}\|f\|_{L^{p}} t^{\theta},
$$

proving (ii). This implies that $\left\|t^{-\theta} K\left(t, f ; H^{1}(\mu), L^{p_{1}}(\mu)\right)\right\|_{L^{\infty}} \leq C_{p}\|f\|_{L^{p}}$, so that $f \in\left[H^{1}(\mu), L^{p_{1}}(\mu)\right]_{\theta, \infty}$ and $\|f\|_{\theta, \infty} \leq C_{p}\|f\|_{L^{p}}$, as required in (iii).

Following closely the proof of [16, Theorem ] we deduce from Lemma 4.5 the following result.

Theorem 4.6. Let $1<p<p_{1} \leq \infty$ and $\theta \in(0,1)$ be such that $\frac{1}{p}=1-\theta+\frac{\theta}{p_{1}}$. Then

$$
\left[H^{1}(\mu), L^{p_{1}}(\mu)\right]_{\theta, p}=L^{p}(\mu) .
$$


4.3. Boundedness of singular integrals on $H^{1}(\mu)$. In this subsection we prove that integral operators whose kernels satisfy a suitable integral Hörmander condition are bounded from $H^{1}(\mu)$ to $L^{1}(\mu)$.

Theorem 4.7. Let $T$ be a linear operator which is bounded on $L^{2}(\mu)$ and admits a locally integrable kernel $K$ off the diagonal that satisfies the condition

$$
\sup _{\tilde{R}} \sup _{y, z \in \tilde{R}} \int_{\left(\tilde{R}^{*}\right)^{c}}|K(x, y)-K(x, z)| d \mu(x)<\infty,
$$

where the supremum is taken over alla Calderón-Zygmund sets $\tilde{R}$ and $\tilde{R}^{*}$ is defined as in Definition 2.10 Then $T$ extends to a bounded operator from $H^{1}(\mu)$ to $L^{1}(\mu)$.

Proof. Using [4.3], by [8, Theorem 1.2] it is easy to prove that the operator $T$ is of weak type $(1,1)$. Then it is enough to show that there exists a constant $C$ such that $\|T a\|_{L^{1}} \leq C$ for any $(1, \infty)$-atom $a$.

Let $a$ be a $(1, \infty)$-atom supported in the Calderón-Zygmund set $\tilde{R}$. Recall that $\tilde{R} \subset B\left(x_{R}, 8 h(\tilde{R})\right)$, and $\tilde{R}^{*}$ denote the dilated set $\{x \in \mathscr{V}: d(x, \tilde{R})<h(\tilde{R}) / 4\}$. We estimate the integral $\int_{\mathscr{V}}|T a| d \mu$.

We first estimate the integral on $\tilde{R}^{*}$ by the Cauchy-Schwarz inequality and the size estimate of the atom:

$$
\begin{aligned}
\int_{R^{*}}|T a| d \mu & \leq\|T a\|_{L^{2}} \mu\left(\tilde{R}^{*}\right)^{1 / 2} \leq C\|\mid T\|\left\|_{L^{2} \rightarrow L^{2}}\right\| a \|_{L^{2}} \mu(\tilde{R})^{1 / 2} \\
& \leq C\|T\| \|_{L^{2} \rightarrow L^{2}} .
\end{aligned}
$$

We consider the integral on the complementary set of $\tilde{R}^{*}$ by using the fact that $a$ has vanishing integral:

$$
\begin{aligned}
\int_{\tilde{R}^{* c}}|T a| d \mu & \leq \int_{\left(\tilde{R}^{*}\right)^{c}}\left|\int_{\tilde{R}} K(x, y) a(y) d \mu(y)\right| d \mu(x) \\
& =\int_{\left(\tilde{R}^{*}\right)^{c}}\left|\int_{\tilde{R}}\left[K(x, y)-K\left(x, x_{R}\right)\right] a(y) d \mu(y)\right| d \mu(x) \\
& \leq \int_{\left(\tilde{R}^{*}\right)^{c}} \int_{\tilde{R}}\left|K(x, y)-K\left(x, x_{R}\right)\right||a(y)| d \mu(y) d \mu(x) \\
& =\int_{\tilde{R}}|a(y)|\left(\int_{\left(\tilde{R}^{*}\right)^{c}}\left|K(x, y)-K\left(x, x_{R}\right)\right| d \mu(x)\right) d \mu(y) \\
& \leq\|a\|_{L^{1}} \sup _{y \in \tilde{R}} \int_{\left(\tilde{R}^{*}\right)^{c}}\left|K(x, y)-K\left(x, x_{R}\right)\right| d \mu(x) \\
& \leq C, \quad
\end{aligned}
$$

as required.

Remark: The previous result applies to singular integral operators associated with the Laplacian $\mathscr{L}$ on the tree defined for every function $f: \mathscr{V} \rightarrow \mathbb{C}$ by

$$
\mathscr{L} f(x)=f(x)-\frac{1}{2 \sqrt{q}} \sum_{y \in \mathscr{V}: d(x, y)=1} q^{\frac{\ell(y)-\ell(x)}{2}} f(y) \quad \forall x \in \mathscr{V} .
$$


The Laplacian $\mathscr{L}$ is bounded on $L^{p}(\mu)$ for every $p \in[1, \infty]$, it is self-adjoint on $L^{2}(\mu)$ and its spectrum on $L^{2}(\mu)$ is [0,2]. Suppose that $M: \mathbb{R} \rightarrow \mathbb{C}$ is bounded supported in $[0,2)$ and satisfies the following Mikhlin-Hörmander condition of order $s>3 / 2$

$$
\sup _{t>0}\left\|\left(D_{t} M\right) \phi\right\|_{W_{2}^{s}}<\infty
$$

for some $\phi \in C_{c}^{\infty}\left(\left[\frac{1}{2}, 4\right]\right), \phi \neq 0$, where $\left(D_{t} M\right)(\lambda)=M(t \lambda)$ and $W_{2}^{s}$ denotes the Sobolev space of order $s$ modelled on $L^{2}([0,2])$. Then the operator $M(\mathscr{L})$ extends to a bounded operator from $H^{1}(\mu)$ to $L^{1}(\mu)$. Indeed, it was shown in [8, Theorem 2.3] that the integral kernel of the operator $H(\mathscr{L})$ satisfies condition 4.3 . For every function $f: \mathscr{V} \rightarrow \mathbb{C}$ we also define the gradient $\nabla f$ by the formula:

$$
(\nabla f)(x)=\sum_{y \in \mathscr{V}: d(x, y)=1}|f(y)-f(x)| \quad \forall x \in \mathscr{V} .
$$

Then the first order Riesz transform $\nabla \mathscr{L}^{-1 / 2}$ extends to a bounded operator from $H^{1}(\mu)$ to $L^{1}(\mu)$. Indeed, it was shown in [8, Theorem 2.3] that the integral kernel of this operator satisfies condition (4.3).

\section{REFERENCES}

[1] L. Arditti. Analysis on weighted homogeneous trees. https://webthesis.biblio.polito.it/8359/, (2018).

[2] J. Bergh, J. Löfström. Interpolation spaces. An introduction. Grundlehren der Mathematischen Wissenschaften, No. 223. Springer-Verlag, Berlin-New York, 1976.

[3] A. Carbonaro, G. Mauceri, S. Meda. $H^{1}$ and $B M O$ for certain locally doubling metric measure spaces. Ann. Sc. Norm. Super. Pisa Cl. Sci. (5) 8 (2009), no. 3, 543-582.

[4] D. Celotto, S. Meda. On the analogueue of the Fefferman-Stein theorem on graphs with the Cheeger property. Ann. Mat. Pura Appl.(4) 197 (2018), no. 5, 1637-1677.

[5] R.R. Coifman, G. Weiss. Extensions of Hardy spaces and their use in analysis. Bull. Amer. Math. Soc. 83 (1977), no. 4, 569-645.

[6] G. B. Folland, E. M. Stein. Hardy spaces on homogeneous groups, Princeton University Press, 1982.

[7] R. Hanks. Interpolation by the real method between $B M O, L^{\alpha}(0<\alpha<\infty)$ and $H^{\alpha}(0<\alpha<\infty)$, Indiana Univ. Math. J. 26 (1977), 679-689.

[8] W. Hebisch, T. Steger. Multipliers and singular integrals on exponential growth groups. Math. Z. 245 (2003), no. 1, $37-61$.

[9] S. Hoory, N. Linial, and A. Widgerson. Expander graphs and their applications. Bull. Amer. Math. Soc. 43 (2006), no. 4, 439-561.

[10] P.W. Jones. Interpolation between Hardy spaces, Conference on harmonic analysis in honor of Antoni Zygmund I, II, (Chicago, 1981), 437-451, Wadsworth Math. Ser., Wadsworth, Belmont, CA, 1983.

[11] J. L. Journé. Calderón-Zygmund Operators, Pseudo-Differential Operators and the Cauchy Integral of Calderón, Lecture Notes in Mathematics 994, Springer-Verlag, 1983.

[12] A. Martini, A. Ottazzi, M. Vallarino. Spectral multipliers for sub-Laplacians on solvable extensions of stratified groups, J. Anal. Math. 136 (2018), 357-397.

[13] J. Peetre. Two observations on a theorem by Coifman, Studia Math. 64 (1979), 191-194.

[14] N.M. Rivière, Y. Sagher. Interpolation between $L^{\infty}$ and $H^{1}$, the real method, J. Funct. Anal. 14 (1973), 401-409.

[15] E. M. Stein. Harmonic Analysis, Princeton University Press, 1993.

[16] M. Vallarino. Spaces $H^{1}$ and $B M O$ on $a x+b$-groups. Collect. Math. 60 (2009), no. 3, 277-295. 
Laura Arditti, Anita Tabacco, Maria Vallarino: Dipartimento di Scienze Matematiche "Giuseppe Luigi Lagrange", Politecnico di Torino, corso Duca degli Abruzzi 24, 10129 Torino, Italy

LAURA.ARDITTI@POLITO.IT, ANITA.TABACCO@POLITO.IT, MARIA.VALLARINO@POLITO.IT 